\title{
Angiographic Assessment of Myocardial Perfusion Using Correlation Analysis
}

\author{
Yu Deuerling-Zheng ${ }^{1}$, Jan Boese ${ }^{1}$, Stephan Achenbach ${ }^{2}$ and Josef Ludwig ${ }^{2}$ \\ ${ }^{1}$ Siemens Medical Solutions, Forchheim, Germany \\ ${ }^{2}$ Cardiology, Friedrich-Alexander-University, Erlangen, Germany \\ Email: yu.deuerling-zheng@siemens.com
}

\begin{abstract}
Although angiography was originally designed as a morphological imaging modality, it is increasingly demanded to retrieve functional information from conventional angiograms. As X-ray angiography provides only $2 \mathrm{D}$ projection images, its most significant advantage over volumetric imaging modalities is its high temporal resolution. Thus the angiographic functional imaging relies mainly on the analysis of the temporal variation of single pixels or local regions. In order to assess the myocardial perfusion, the myocardium has to be recognized at first. This can be solved similarly with analysis of the time-intensity curves. Compared to most approaches in which the characteristic of the time-intensity curves are classified, our proposed method based on correlation analysis has the advantage of using all the sampled data points so that it is more robust against noise or outliers. As the correlation coefficient considers only the colinearity of two curves, we add the information about the amplitude and the numerical range of the curves, which further improves the recognition of the myocardium.
\end{abstract}

\section{Introduction}

Percutaneous coronary intervention (PCI) - also known as coronary angioplasty - is an established treatment strategy for acute myocardial infarction (AMI). The goal is to open the occluded infarct artery to facilitate prompt reperfusion of ischemic myocardium. Since the introduction of PCI, much effort has been done to retrieve functional information out of angiograms for the assessment of reperfusion.

Due to the high concentration of the contrast agent in the arteries, initial efforts were focused on measuring the blood flow in the coronary arteries. However, it has been shown that the opening of the stenosis in the epicardial artery does not necessarily lead to improvement of the microvascular circulation. To assess the perfusion directly at the capillary level, two dedicated measures are proposed: TIMI Myocardial Perfusion Grade (TMPG) [1] and Myocardial Blush Grade (MBG) [2]. TMPG emphasizes the dynamic of the contrast enhancement while MBG characterizes the contrast enhancement rather by its density or brightness. 
Clinical trials have shown that both TMPG and MBG are independent predictors for the long-term outcome of patients with AMI $[1,2]$. They correlate well with other non-angiographic measures and the mortality. However, these two measures are assessed nowadays only visually in a few core labs worldwide. An automated and objective tool for angiographic assessment of myocardial perfusion has recently become the research of interest for both physicians and computer scientists.

\section{State of the art}

Recognition of the myocardium is the essential prerequisite for the (re-)perfusion assessment. While the coronary arteries filled with dye show normally high contrast and sharp edges, the myocardium is usually rather low-contrasted with the ground-glass appearance ("blush") without clear boundaries. Therefore, recognition of the myocardium remains a challenging task for angiographic perfusion assessment.

Principally, the efforts for the recognition of myocardium can be divided into two categories:

1. Morphological approach: outline the region of interest (ROI) for the blush out of a single frame based on morphological analysis and track the ROI in the consecutive frames [3]. The drawback of this approach is that the blush must show sufficient contrast to ensure its differentiation from other structures.

2. Temporal approach: retrieve the time-intensity curve for each pixel or local region and differentiate the myocardium according to the characteristics of the time-intensity curves, such as the maximum amplitude and the time to maximum amplitude $[4,5]$. This approach is preferable because it directly analyzes the temporal information, which emphasizes the basic advantage of angiography. However, it is also inherently sensitive to object misalignment between the frames, which is unavoidable in coronary angiograms due to respiratory and cardiac motion. Motion compensation is thus one of the main research interests in this field nowadays [4, 5].

\section{Methods - correlation analysis}

Structures in angiographic series can be differentiated from each other by classifying the features of their time-intensity curves (Fig. 1). The most commonly used features are the maximum amplitude and the time to maximum amplitude. This requires that the features are extracted in a robust manner, for instance with previous elimination of outliers, which is however in practice computationally highly intensive. In this work, an approach based on correlation analysis of the time-intensity curves is presented. This approach has been applied to separate pulmonary arteries and veins in 3D MR angiography [6]. After manually selecting a region of interest (ROI) to define the reference, the correlation 
Fig. 1. Time-intensity curves of contrast-enhanced coronary artery, myocardium and non contrast-enhanced background structures

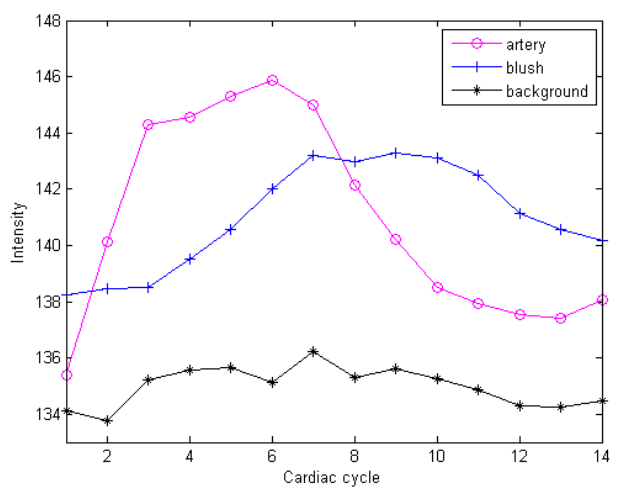

coefficient between time-intensity curve of the reference and those of all the local regions is computed. This is then used as a similarity measure between the reference and local regions. This approach has the advantage by using all the sampled data points in the time-intensity curve, which makes it less sensitive against outliers.

Let $X$ and $Y$ be the vectors containing the values of two time-intensity curves, $\bar{x}$ and $\bar{y}$ the mean of $X$ and $Y$, respectively. The correlation coefficient between $X$ and $Y$ can be computed as in (Eq. 1). $c(X, Y)$ assumes values between -1 and +1 with the magnitude indicating the strength of the dependence. The correlation is positive if both $X$ and $Y$ increase or decrease together, and negative if $Y$ decreases when $X$ increases. If $X$ and $Y$ are said to be independent or uncorrelated, $c(X, Y)$ disappears

$$
c(X, Y)=\frac{\sum_{i=1}^{n}\left(x_{i}-\bar{x}\right)\left(y_{i}-\bar{y}\right)}{\sqrt{\sum_{i=1}^{n}\left(x_{i}-\bar{x}\right)^{2} \sum_{i=1}^{n}\left(y_{i}-\bar{y}\right)^{2}}}
$$

The correlation coefficient is normalized, i.e., it measures the similarity between $X$ and $Y$ only by considering their colinearity, with the magnitude of $X$ and $Y$ being ignored. Moreover, $X$ and $Y$ are shifted to the zero mean so that the actual numerical range is not considered either. However, the time-intensity curves of different tissues differ not only in the phase but also in the amplitude and the value range of the intensity. For the purpose of an accurate discrimination, it is important to take into account as many as possible discriminating features, for instance as following

$$
F_{a}=\frac{\min \left(A_{x}, A_{y}\right)}{\max \left(A_{x}, A_{y}\right)} \quad F_{g}=\frac{\min (\bar{x}, \bar{y})}{\max (\overline{x, y})} \quad c^{\prime}(X, Y)=F_{a}^{p} \cdot F_{g}^{q} \cdot c(X, Y)
$$


where $A_{x}$ and $A_{y}$ are the amplitude (difference between the maximal and minimal intensity) of $X$ and $Y$, respectively. Both $F_{a}$ and $F_{g}$ assume values in $(0,1]$ with $p$ and $q$ as weighting exponents.

\section{Results}

The angiographic data sets used in this work are acquired with a C-Arm system (AXIOM Artis, Siemens Medical Solutions). Analyzing time-intensity curves of single pixels or local regions assumes that the frames of an angiographic series are spatially aligned. To avoid respiratory motion, angiograms are acquired with patient breath holding. To avoid cardiac motion, a retrospective ECG-gating is performed to select only the frames corresponding to a certain cardiac phase. The resulting time-intensity curves contain normally 10-20 data points.

The correlation coefficient illustrated in Section 3 serves as a similarity measure between two time-intensity curves. To outline the myocardium, a reference curve needs to be determined. This can be done either empirically out of a sufficient large number of data sets, or with user interaction by mouse clicking in the region of the myocardium. The latter is applied in this work since it can adjust variations between different acquisitions with only minimal user interaction. The correlation coefficient between the time-intensity curve of the reference and those of all the local regions is computed (which returns the so-called correlation coefficient map). The regions where the correlation coefficient is larger than a certain threshold are classified as myocardium.

Our experiments on 5 angiographic sequences of the right coronary artery show that the myocardium can be recognized with the proposed approach. Fig. 2 shows the result of an example. The contrast enhancement in the original angiogram is hardly visible while the correlation map shows a rather clear, connected bright area indicating high correlation with the reference curve. The myocardium could be recognized after thresholding of the correlation coefficient. It can also be seen that there are sporadically distributed regions in the background which are falsely recognized as myocardium.

\section{Discussion}

As mentioned in Section 3, correlation analysis has the advantage of using all the data points in the time-intensity curve, which is more robust against noise and outliers. Outliers in the time-intensity curve occur quite often because motion during coronary angiography can usually not be avoided or corrected completely by breath-holding and ECG-gating. Therefore, to reduce noise or outliers, the time-intensity curve is retrieved not for single pixels but for a local region of pixels, with the mean of the local region as one data point in the curve. Dedicated approach for motion compensation is currently under working process. Similarly, for the sake of a reliable reference curve, it is to be recommended that the reference defined by a sufficient large region of interest. 
Fig. 2. (a) A frame of the original angiogram acquired after contrast injection in the right coronary artery (RCA). The related myocardium is slightly darker than the surrounding structures, which is however hardly visible. (b) The correlation coefficient map showing local correlations with a reference region selected within the myocardium, bright for high correlation and dark for low correlation. (c) The recognized myocardium: regions with a correlation coefficient larger than a threshold of 0.5 are marked with red blocks

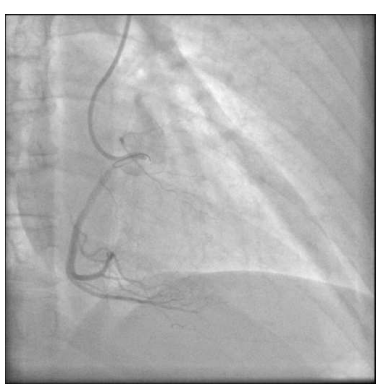

(a)

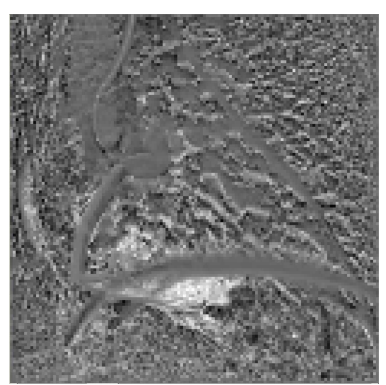

(b)

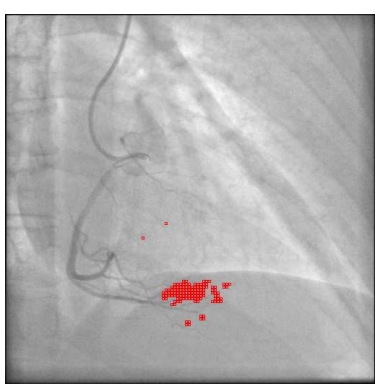

(c)

We have shown that correlation analysis is a feasible approach to recognize contrast-enhanced myocardium in angiograms. This is the first step for the further perfusion assessment of the myocardium. Correlation analysis can be applied to assess the myocardial perfusion as well. As MBG emphasizes the perfusion mainly by the strength of the blush which corresponds to the amplitude of the time-intensity curve, correlation analysis is more advantageous for the assessment with TMPG. Nevertheless, it should be pointed out that correlation analysis can not be used to recognize static objects whose time-intensity curve is flat. In case of $\mathrm{TMPG}=0$ where no contrast agent enters the myocardium, special consideration must be taken into account.

\section{References}

1. Gibson C, et al. Coronary and myocardial angiography: Angiographic assessment of both epicardial and myocardial perfusion. Circulation 2004;109(25):3096-3125.

2. van't Hof A, et al. Angiographic assessment of myocardial reperfusion in patients treated with primary angioplasty for acute myocardial infarction: Myocardial blush grade. Circulation 1998;97(23):2302-2306.

3. Condurache A, et al. User-defined ROI tracking of the myocardial blush grade. Procs IEEE Southwest Symposium on Image Analysis and Interpretation 2006; 66-70.

4. Esbacher M, Dickhaus H, Kücherer H. Computergestützte Auswertung koronarangiographischer Bildfolgen hinsichtlich des Myocardialen Blushgrades. Procs BVM $2006 ; 241-245$.

5. Malsch U, Dickhaus H, Kücherer H. Quantitative Analyse von Koronarangiographischen Bildfolgen zur Bestimmung der Myokardperfusion. Procs BVM 2003; 81-85.

6. Bock M, et al. Separation of arteries and veins in 3D MR angiography using correlation analysis. Magn Reson Med 2000; 481-487. 